\title{
Myasthenia with interesting systemic associations - A case series
}

\section{Muthu Krishnan Vallinayagam1,", Datta Gulnar Pandian², Vasudev Anand Rao ${ }^{3}$}

${ }^{1}$ Assistant Professor, Dept. of Ophthalmology, Sri Lakshmi Narayana Institute of Medical Sciences, Puducherry, ${ }^{2}$ Assistant Professor, ${ }^{3}$ Professor, Dept. of Ophthalmology, Sri Venkateshwaraa Medical College Hospital, Puducherry, India

*Corresponding Author:

Email: muthu2308@yahoo.co.in

\begin{abstract}
Myasthenia gravis (MG) is an autoimmune disorder with a highly variable clinical expression. Ocular symptoms are the presenting features in $40 \%$ patients but MG is restricted to ocular muscles in $15 \%$. Myasthenia gravis is often associated with other autoimmune diseases. Case details of ten myasthenic patients visiting ophthalmology department over two years in a tertiary care hospital were analyzed. Five of them had interesting systemic associations. One patient presented with coexisting thyroid ophthalmopathy and ocular myasthenia. Because of the clinical overlap of ocular manifestations in myasthenia and Graves' disease, one of the two conditions may be missed. Two patients had thymic mass. Presence of thymoma and prognostic implications are highlighted. One patient had thymic cyst, pemphigus and diabetes mellitus. This assortment of autoimmune conditions has not been reported in literature. One patient had associated Parkinson's disease. Myasthenia gravis presents with a highly variable clinical spectrum and is associated with a cluster of autoimmune diseases. This unfortunate association is not widely appreciated. This report highlights the importance of detecting systemic associations of MG. The clinical relevance of these associations in diagnosis and management of myasthenia are discussed.
\end{abstract}

Keywords: Myasthenia gravis, Pemphigus, Thyroid ophthalmopathy, Thymoma.

\section{Introduction}

Myasthenia gravis (MG) is an autoimmune neuromuscular disorder with a highly variable clinical expression. ${ }^{1}$ Ocular symptoms are the presenting features in $40 \%$ patients but MG is restricted to the ocular muscles in $15 \%$ of cases. Thymic involvement in the form of thymoma or thymic hyperplasia is seen in 70- $90 \%$ MG. $^{2}$ MG coexists with autoimmune diseases such as Hashimoto's thyroiditis, Graves' disease, polymyositis, systemic lupus erythematosus, diabetes mellitus, rheumatoid arthritis and pemphigus vulgaris. ${ }^{1,3}$ This report highlights the importance of detecting systemic associations in MG, which greatly influence the disease course and have crucial implications in management.

\section{Case Series}

Case details of myasthenic patients presenting to ophthalmology clinic over the past two years in a tertiary care hospital were analyzed. Ethical approval was given by the institutional ethics committee. Ten myasthenic patients with ocular complaints are discussed in this series. (Table 1)

Table 1: Clinical features, investigations and associations in a series of 10 patients with myasthenia gravis

\begin{tabular}{|l|c|c|c|c|c|c|c|}
\hline S. No & Age/Sex & Ptosis & Ophthalmoplegia & $\begin{array}{c}\text { Ice-pack } \\
\text { Test }\end{array}$ & $\begin{array}{c}\text { Neostig-mine } \\
\text { test }\end{array}$ & $\begin{array}{c}\text { SF- } \\
\text { EMG }\end{array}$ & Systemic Association \\
\hline 1 & $10 / \mathrm{M}$ & + & + & + & + & + & Nil \\
\hline 2 & $50 / \mathrm{F}$ & + & + & - & + & + & $\begin{array}{c}\text { Systemic MG, Thymic } \\
\text { cyst, Pemphigus, DM }\end{array}$ \\
\hline 3 & $16 / \mathrm{M}$ & + & + & + & + & + & Nil \\
\hline 4 & $50 / \mathrm{F}$ & + & + & + & + & + & Systemic MG Thymoma \\
\hline 5 & $60 / \mathrm{M}$ & + & + & + & + & + & Nil \\
\hline 6 & $55 / \mathrm{F}$ & + & + & + & + & + & Nil \\
\hline 7 & $67 / \mathrm{M}$ & + & + & + & + & + & Parkinson's disease \\
\hline 8 & $58 / \mathrm{F}$ & + & - & + & + & Thymoma \\
& & & + & + & + & + & Hyperthyroidism Thyroid \\
eye disease
\end{tabular}

*SF-EMG: Single fiber electromyogram*M - Male

*DM: Diabetes mellitus*F - Female

*MG: Myasthenia gravis

Three patients had systemic MG while in others, the disease was limited to ocular involvement. All patients had ptosis and extraocular movement restriction. Clinical diagnosis was aided by transient improvement of ptosis on ice-pack test. MG was confirmed by positive neostigmine test and single fibre electromyogram. Chest x-ray and computed 
tomography of chest for thymoma was done. Five of them had interesting systemic associations.

One patient presented with coexisting thyroid ophthalmopathy and ocular myasthenia. He presented with bilateral proptosis with ptosis. Systemic examination revealed a thyroid swelling and features of hyperthyroidism. Ice-pack test was positive (Fig. 1Coexisting ocular myasthenia and Graves' disease \& Fig. 2 - Improvement of ptosis on ice-pack test). Forced duction test and neostigmine test were positive. Thyroid function tests were abnormal. There was a good response to medical treatment with propranolol, neomercazole and pyridostigmine.

Two patients had associated thymoma diagnosed on CT scan (Fig. 3- CT scan showing mediastinal mass suggestive of thymoma) and underwent thymectomy followed by histopathology of excised mass (Fig. 4Histopathological slide of thymoma).

A female patient presented with thymic cyst, pemphigus and diabetes mellitus. She was treated with cyclophosphamide and dexamethasone pulse therapy, oral hypoglycemics and thymic cyst excision with thymectomy. Myasthenic symptoms were controlled with pyridostigmine and oral prednisolone.

Another patient had associated Parkinson's disease and was symptomatically better with pyridostigmine and levodopa-carbidopa combination.

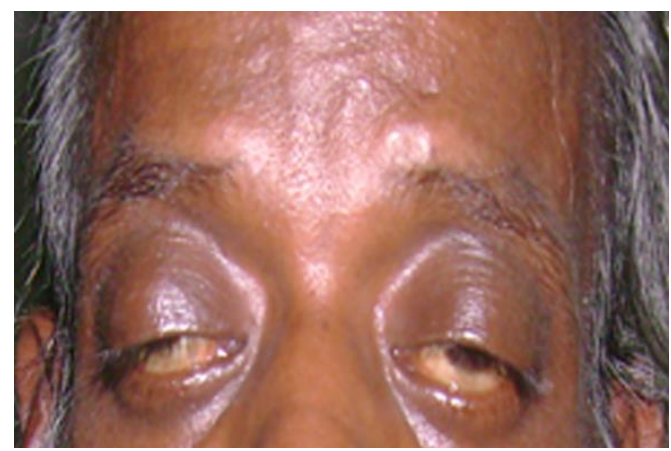

Fig. 1: Coexisting myasthenia gravis and graves' disease

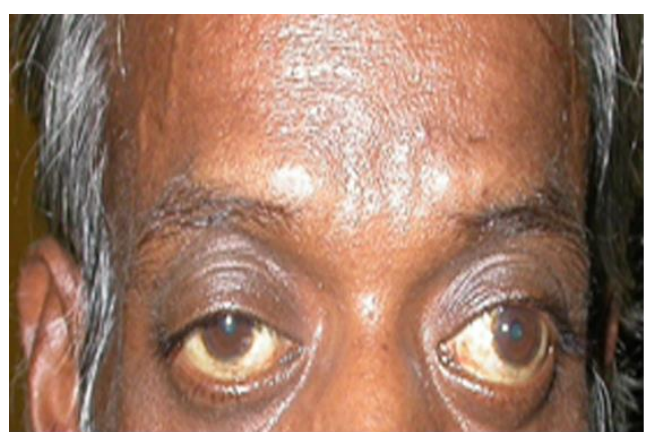

Fig. 2: Improvement of ptosis on ice-pack test

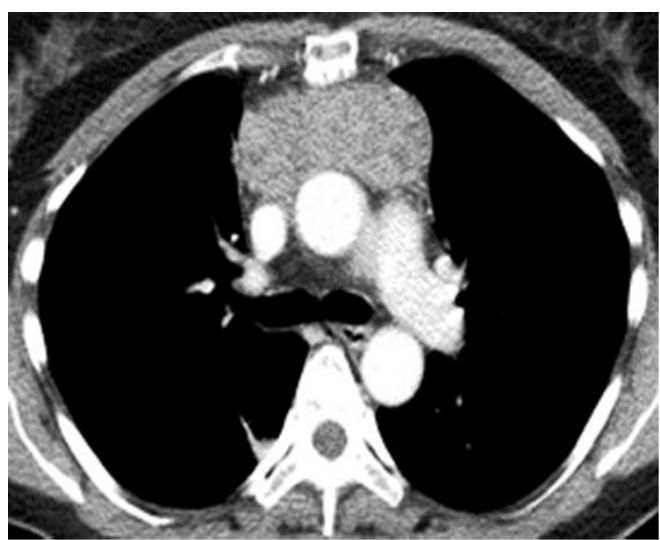

Fig. 3: CT scan of thymoma

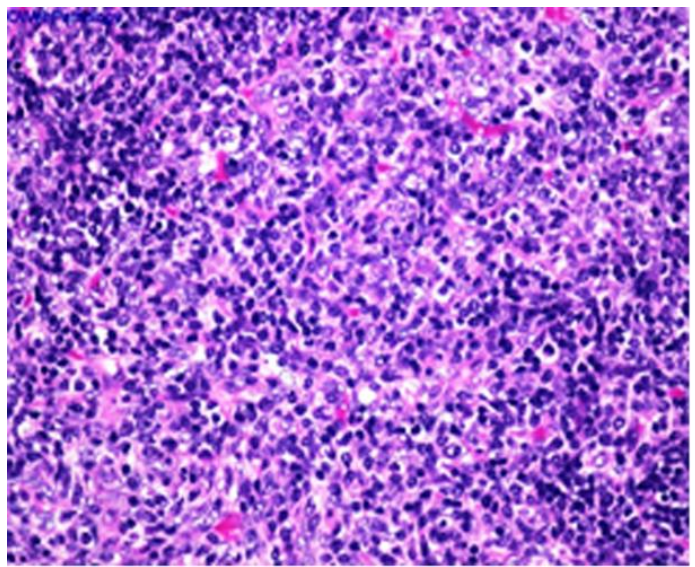

Fig. 4: Histopathology of thymoma

\section{Discussion}

Myasthenia gravis (MG) is an autoimmune disorder with myriad of manifestations ranging from localized ocular myasthenia to generalized MG. ${ }^{1}$ Ptosis and diplopia are the presenting features in $40 \%$ MG but the disease is restricted to ocular muscles in $15 \%$. $^{2}$

Autoimmune thyroid disease occurs with a higher prevalence in myasthenia. ${ }^{1,4}$ This association is consistent with a genetic predisposition for autoimmune disease. Our case presented simultaneously with MG and Graves' disease. MG occurs mostly subsequent to Graves' (40-50\%), but can precede Graves' (30-35\%) or occur simultaneously $(20-25 \%) .{ }^{1}$ Immunological cross-reactivity against epitopes or autoantigens shared by the thyroid and ocular muscles is speculated to be the basis for this association. ${ }^{1,4}$ Autoimmune thyroid eye disease occurs in 5-10\% MG whereas MG is seen only in $0.2 \%$ of thyroid disease. ${ }^{2-4}$ Graves' is frequently associated with ocular myasthenia rather than its systemic form. ${ }^{2}$ The clinical overlap of both entities can pose a diagnostic challenge unless high index of vigilance is entertained. Presence of ptosis in a patient with thyroid eye disease should arouse suspicion of coexisting MG. ${ }^{1,4}$ MG can influence the medical (beta blockers, benzodiazepines) or surgical treatment (preoperative neuromuscular blockers) of Graves' by leading to myasthenic crisis if adequate precautions are 
not taken. ${ }^{1}$ Conversely, restoration of euthyroid state may alleviate myasthenic symptoms. ${ }^{4} \mathrm{MG}$ and thyroid eye disease are infrequently associated with thymic abnormalities suggesting the role of thymus in immunopathogenesis of both the conditions. ${ }^{1-4}$

Thymus is involved in $70-90 \% \mathrm{MG}$ as thymic hyperplasia or thymoma. Thymoma complicates 10 $15 \%$ of $\mathrm{MG}$ and $\mathrm{MG}$ is speculated to be a paraneoplastic disease caused by the presence of thymoma. ${ }^{2,6}$ Thymoma is an anterior mediastinal tumor which peaks in fifth and sixth decades with a preponderance in males. Both thymoma patients in our series were females above 50 years. Thymectomy is absolutely indicated in thymoma due to locally invasive nature. ${ }^{5,6}$ Thymectomy results in remission of myasthenic symptoms in $80 \%$ cases. . $^{1,25,6}$

A female patient presented with myasthenia, thymic cyst and pemphigus, a constellation of disorders not reported in literature. Pemphigus is an immunologically mediated disease associated with thymic neoplasm. Thymectomy is reported to have a variable effect on thymoma related pemphigus. ${ }^{7}$ The concurrence of autoimmune diseases may involve the failure of a subgroup of thymic dependent lymphocytes to suppress underlying autoimmune phenomena. ${ }^{8}$ Thymic cyst is rarely reported with MG and it is unlikely that surgical resection would influence the natural history of MG. ${ }^{2}$ Our patient with thymic cyst was subjected to thymectomy to rule out remote possibilities of thymic carcinoma. Subsequently there was no spontaneous remission of MG or pemphigus.

A 67 year old patient of Parkinson's disease, developed MG. Occurrence of myasthenia is rare in elderly. He was treated with levodopa-carbidopa and pyridostigmine. Myasthenia and Parkinson's disease have a confluence of common clinical features like 'dropped head', gait disturbance, speech and swallowing difficulty and weakness of facial expression..$^{9}$ Hence awareness of the co-morbidity is important for the prompt appreciation. Though the association is rare, few reports suggest a possible immune link between MG and Parkinson's. There is a report on trihexiphenydyl induced myasthenia and another on pyridostigmine induced Parkinson's disease. ${ }^{9,10}$ Since an immune mechanism for Parkinson's has not yet been elucidated, further research is required to prove that the association between MG and Parkinson's is other than purely fortuitous.

\section{Conclusion}

Autoimmune diseases tend to be clustered together, more often than can be ascribed to chance. The unfortunate association between $\mathrm{MG}$ and other autoimmune disorders is not widely appreciated. This report intends to highlight the importance of detecting systemic associations in MG which may have crucial implications in disease course. Since majority of myasthenic patients present first to the ophthalmologist, it is imperative to stay aware of these systemic associations for early diagnosis and appropriate management. Further research is called for into the strict surveillance, early recognition and effective management of coexisting autoimmune disorders.

\section{References}

1. Pouye A, Dia DG, Ndongo S, Faye A, Sakho ND and Ndiaye FSD. Grave's disease associated with myasthenia gravis: a case report. Intern Med Inside. 2014;2:1-4. doi: 10.7243/2052-6954-2-4.

2. Peacey SR, Belchetz E. Graves' disease: associated ocular myasthenia gravis and a thymic cyst. $J R$ Soc Med. 1993;86(5):297-298.

3. Huai-Hua Y, Yung-Wei T, Chi-Chiang Y, Jai-Nien T. Myasthenia Gravis with Thymoma and Coexistent Central Hypothyroidism. J Chin Med Assoc. 2009;72(2):91-93.

4. Chhabra S, Pruthvi BC. Ocular myasthenia gravis in a setting of thyrotoxicosis. Ind J Endocr Metab. 2013;17(2):341-343.

5. Manjiri K, Swami RM. Type A Thymoma with Generalised Myasthenia Gravis. Indian J Chest Dis Allied Sci. 2011;53(4):233-235.

6. Romi F. Thymoma in Myasthenia Gravis: From Diagnosis to Treatment. J Autoimmune Dis 2011, 2011:474512. doi: 10.4061/2011/474512.

7. Takeshita K, Amano M, Shimizu T, Oyamada Y, Abiko Y, Kobayashi K et al. Thymoma with Pemphigus Foliaceus. Intern Med. 2000;39(9):742- 747.

8. John CM, Richard LD, Thomas TP. Pemphigus and Myasthenia Gravis. Arch Dermatol. 1975;111(10):13341339.

9. Levin N, Karussis D, Abramsky O. Parkinson's disease associated with myasthenia gravis. J Neurol. 2003;250(6):766-767.

10. Kao KP, Kwan SY, Lin KP, Chang YC. Coexistence of Parkinson's disease and myasthenia gravis: a case report. Clin Neurol Neurosurg. 1993;95(2):137-139.

How to cite this article: Vallinayagam MK, Pandian DG, Rao VA. Myasthenia with interesting systemic associations - A case series. Ind J Clin Exp Ophthalmol. 2018;4(3):430-432. 